\title{
Isolement de souches HIV-1 résistantes à I'AZT : limites des analogues de nucléosides dans la chimiothérapie du SIDA
}

Le traitement du SIDA par la 3'-azido-3'désoxythymidine (AZT) est le seul dont l'efficacité ait, à ce jour, été prouvée. Malheureusement, il entraîne, chez au moins $75 \%$ des malades, l'apparition de souches résistantes de HIV-1. La transcriptase inverse de ces souches résistantes ne semble pas modifiée cinétiquement lors de tests in vitro, alors que la sensibilité des virus à l'AZT est très diminuée. Les virus résistants sont moins infectieux, mais plus cytopathiques que leur contrepartie sensible. Leur génome semble spécifiquement muté au niveau du gène pol. La résistance à l'AZT peut être accompagnée d'une résistance croisée à d'autres substances, fait à prendre en compte pour l'institution d'une bi-chimiothérapie évitant ou retardant l'apparition de souches résistantes.

\section{Ronald Rooke Michel Tremblay Claude Lalande Michael A. Parniak Marc A. Wainberg}

\section{ADRESSES}

R. Rooke : chargé de cours à l'université Mc Gill. M. Tremblay: chargé de cours à l'universilé Mc Gill. C. Lalande : chargé de cours à l'université Mc Gill. M.A. Parniak : professeur adjoint à l'universilé Mc Gill. Institut Lady Davis, Hôpital général juif, département de microbiologie et d'immunologie, et centre SIDA McGill, université McGill, Montréal, Québec, Canada. M.A. Wainberg : professeur tilulaire el direcleur du centre SIDA de l'universilé Mc Gill. Hôpital général

juif, 3755, chemin Côte-Sainte-Catherine,
Montréal, Québec, H3T 1E2, Canada. 'agent étiologique du SIDA, le virus de l'immunodéficience humaine de type 1 (HIV-1), est le dernier d'une longue série de virus qui provoquent chez l'homme une suppression de la réponse immunitaire. Dès 1908, von Pirquet [1] démontrait que plusieurs patients infectés par le virus de la rubéole souffraient de profonds dérèglements du système immunitaire. Depuis lors, il a été montré que de nombreuses infections virales sont la cause d'une réponse immunitaire anormale tant chez les animaux que chez les humains [2]. Les virus qui peuvent provoquer une immunodéficience proviennent de familles très disparates : certains virus à $A R N$ tel le virus influenza, des virus à $\mathrm{ADN}$ comme le cytomégalovirus (CMV), le virus Epstein-Barr (EBV), les virus herpes simplex (HSV-1 et HSV-2) et, plus récemment identifiés, plusieurs rétrovirus [3].
Toutefois, comparé aux autres immunodéficiences d'origine infectieuse, le SIDA soulève des problèmes particuliers. Tout d'abord, cette maladie est jusqu'à maintenant incurable et ses symptômes sont pour la plupart irréversibles. L'immunosuppression causée par le HIV-1 est très importante : les personnes atteintes sont sensibles à une vaste gamme d'infections opportunistes et de néoplasies, causant la mort à plus ou moins brève échéance [4]. De plus, le nombre d'individus infectés par le HIV-1 est en constante progression dans plusieurs parties du globe [5]. Finalement, le rétrovirus responsable de l'infection subit une dérive génétique très importante, rendant complexe et ardu le développement d'un vaccin [6].

Pour toutes ces raisons, il est essentiel que des stratégies efficaces de lutte contre le HIV-1 soient mises au point. Outre la prévention et la vac- 
cination, qui visent à protéger la population non atteinte, il apparaît évident que la seule façon de contrecarrer efficacement la réplication et les effets néfastes du HIV-1 réside en l'utilisation de plusieurs produits qui s'attaqueront simultanément à plusieurs étapes du cycle de réplication du virus [7]. Cette approche thérapeutique est particulièrement importante : en effet, de récents résultats, obtenus dans notre laboratoire et ailleurs, montrent que la première chimiothérapie approuvée pour une utilisation clinique à grande échelle contre le HIV-1, à savoir le traitement par la zidovudine (3'-azido-3'désoxythymidine ou AZT), peut conduire à la sélection de souches virales résistantes à cette drogue chez la majorité des patients traités [8,9].

Étant déjà impliqué dans l'isolement du HIV-1 à partir du sang de patients traités par la zidovudine, il nous apparut essentiel de déterminer si les souches virales présentes chez ces individus avaient la capacité d'infecter des cellules cibles en présence d'une concentration inhibitrice de drogue. Ayant obtenu de tels variants du HIV-1, il était crucial d'évaluer leur virulence, leur potentiel cytopathologique ainsi que leur sensibilité à d'autres agents chimiothérapeutiques. Nous nous proposons donc de faire le point sur la recherche menée par notre groupe, ainsi que par d'autres équipes, sur les souches du HIV-1 résistantes à l'AZT et sur leur importance dans les stratégies anti-rétrovirales. Nous présenterons d'abord un bref aperçu du cycle de réplication du virus, ce qui nous permettra ensuite de situer et de comprendre chacune des stratégies spécifiques de la chimiothérapie. Finalement, nous discuterons de la résistance aux chimiothérapies et de son importance.

\section{Le cycle de reproduction du HIV-1}

Interaction du virus avec les récepteurs cellulaires. Comme pour tous les rétrovirus connus, le cycle de reproduction du HIV-1 commence par le contact entre le virion et un récepteur spécifique présent à la surface des cellules hôtes. Dans le cas du HIV-1, la glycoprotéine gp120 de l'enveloppe du virion interagit avec l'antigène (ag) de surface CD4 [10]. De nombreux types cellulaires possèdent à leur surface le récepteur CD4 : les lymphocytes T4-auxiliaires, les macrophages, les lymphocytes B, les cellules endothéliales, dendritiques, nerveuses et cérébrales [11, 12]. Les lymphocytes T4-auxiliaires, qui orchestrent la réponse immunitaire, constituent la population cellulaire possédant la plus haute concentration de CD4 à leur surface. Ces cellules sont ainsi rendues hautement sensibles à l'infection et à la destruction par le HIV-1. Les effets cytopathogéniques causés par le virus sur les autres types cellulaires exprimant le CD4 semblent nettement moins prononcés et pourraient être reliés à la plus faible quantité de récepteur à leur surface.

Entrée du virus dans la cellule et conversion de l'ARN viral en ADN. Après la fixation initiale au récepteur CD4 via la molécule gp120, c'est à l'aide de la glycoprotéine transmembranaire de l'enveloppe du virus, la gp41, que fusionnent l'enveloppe virale et la membrane cellulaire [13]. Le virion est alors relâché dans le cytoplasme sous la forme d'un complexe nucléoprotéique. Le génome viral est formé de deux châ̂nes identiques d'ARN monocaténaire, encapsidées dans le nucléocapside du virion et associées à une ADN-polymérase dépendante de l'ARN, la transcriptase inverse (TI). Le rôle de cette enzyme est de convertir l'ARN viral en ADN bicaténaire qui migrera jusque dans le noyau cellulaire, où il s'intégrera au hasard dans le génome de la cellule hôte. L'ADN ainsi intégré, que l'on appelle ADN proviral ou provirus, ne peut être distingué de l'ADN cellulaire et sera donc répliqué et exprimé au même titre qu'un gène de la cellule hôte. La TI, particulière aux rétrovirus, constitue ainsi une cible de choix pour une chimiothérapie spécifiquement dirigée contre ces virus.

Expression de l'ARN viral et des protéines virales. L'expression des gènes du provirus s'effectue en utilisant la machinerie cellulaire. Certaines protéines virales sont synthétisées sous forme de précurseur de poids moléculaire élevé et ne deviennent fonctionnelles que lorsque le précurseur est scindé par la protéase virale.

Assemblage et formation des virions. Les virions sont assemblés au niveau de la membrane cellulaire où une forte concentration de protéines virales est alors présente. Le complexe nucléoprotéique, formé par deux copies d'ARN simple brin et de nombreuses protéines virales, est enveloppé par la membrane cellulaire et relâché dans l'environnement par bourgeonnement.

\section{Stratégies chimiothérapeutiques}

Presque toutes les stratégies thérapeutiques anti-HIV-1 en développement ou déjà utilisées peuvent être regroupées en deux classes selon leur mécanisme d'action : (1) les chimiothérapies anti-rétrovirales directes qui tentent d'arrêter le cycle de réplication du virus à une étape ou à une autre; (2) les immunomodulateurs dont le but est d'intensifier ou de restaurer les défenses immunitaires du patient [14]. Le chapitre suivant décrit le mode d'action de certains produits faisant partie de la première classe d'agents thérapeutiques. Il est important de considérer que l'utilisation de combinaisons d'agents qui inhibent la réplication virale à des stades différents du cycle pourrait avoir un effet synergique sur celle-ci et limiter l'apparition de souches virales résistantes aux produits administrés simultanément.

Adsorption et pénétration. Bon nombre de chercheurs ont tenté de bloquer l'interaction de la gp120 virale avec le récepteur cellulaire CD4. L'approche qui, jusqu'à présent, a été la plus fructueuse est fondée sur l'usage d'une forme soluble de CD4 (sCD4) produite par génie génétique $[15,16]$. Le sCD4, incubé avec du HIV-1, se lie à la gp120 de l'enveloppe du virion et bloque ainsi l'interaction de ces particules virales avec le CD4 présent à la surface des cellules cibles. D'autres substances inhibitrices de l'adsorption virale sont des polymères polyanioniques tel le sulfate de dextran. L'action de ces molécules est liée à leur forte charge négative qui empêcherait l'interaction gp120:CD4. Ces approches sont toutefois limitées car 


\section{RÉFÉRENCES}

1. Von Pirquet C. Das Verhalten der kutanen tuberculin-Reaktion während der Masern. Deulsche Medizin Wochensch 1908 ; $34: 1297-310$

2. Wainberg MA, Mills EL. Mechanisms of virus-induced immune suppression. Can Med Assn 1985; 132 : 1261-7.

3. Gardner MB, Lucin PA. Animal models of AIDS. FASEB 1989; 3 : 2593-606.

4. Gallo RC, Salahuddin SZ, Popovic M. Frequent detection and isolation of cytopathic retroviruses (HTLV-III) from patients with AIDS and at risk for AIDS. Science $1984 ; 244$ : 500-3.

5. Piot P, Plummer FA, Mhalu FS, el al. AIDS : an international perspective. Science 1988 ; 239 : $573-9$.

6. Magasiny S, Spina B, Barré-Sinoussi F, Chermann JC. Genomic variability of selected LAV-related AIDS retroviruses. AIDS Res 1986 ; 2 : 19-30.

7. Girard M. Vaccins contre le SIDA espoirs et réalités. médecine/sciences $1989 ; 5$ : 152-8.

8. Rooke R, Tremblay M, Soudeyns H, et al. Isolation of drug-resistant variants of HIV-1 from patients on long-term zidovudine therapy. $A I D S 1989 ; 3: 411-5$

9. Larder BA, Darby G, Richman DD HIV with reduced sensitivity to zidovudine (AZT) isolated during prolonged therapy. Science 1989 ; 243 : 1731-4.

10. Klatzman D, Champagne E, Chamaret S, el al. T-lymphocyte T4 molecule behaves as the receptor for human retrovirus LAV. Nalure 1984; 312 : 767-8.

11. Gray F. Les lésions du système nerveux central au cours du SIDA. médecine/sciences $1989 ; 5: 145-51$.

12. Gendelman HE, Orenstein JM, Bach LM, et al. The macrophage in the persistence and pathogenesis of HIV infection. AIDS 1989 ; 3 : 475-96.

13. Kowalski M, Poeitz J, Basiripour L, et al. Functional regions of the envelope glycoprotein of HIV-1. Science 1987; 237 . 1351-5.

14. Darlix JL. Nouvelles perspectives en chimiothérapie anti-rétrovirale. médecine/sciences 1989 ; 5 : 213-9.

15. Deen KC, McDougal JS, Inacker $R$ et al. Soluble CD4 form of CD4 (+4) protein inhibits AIDS virus infection. Nalure $1988 ; 331: 82-4$

16. Trunecker JA, Luke W, Kanjaleinen K. Soluble CD4 molecules neutralize human immunodeficiency virus type 1 . elles ne peuvent qu'empêcher l'infection de cellules susceptibles. Elles ne permettent donc ni d'éliminer les cellules déjà infectées ni de prévenir la production de nouveaux virions.

La transcriptase inverse (TI). Cette enzyme est essentielle à la réplication des rétrovirus et n'est pas impliquée dans les fonctions cellulaires normales. Cela en fait donc une cible de choix pour la chimiothérapie antirétrovirale. Un des inhibiteurs de l'activité de la TI est l'AZT (3'azido-3'-désoxythymidine ou zidovudine). Cet agent, le plus souvent utilisé en chimiothérapie anti-HIV-1, est un analogue de la thymidine et agit comme inhibiteur compétitif de la TI [17]. Lorsqu'il est incorporé dans l'ADN viral, l'AZT - qui possède un radical $\mathrm{N}_{3}$ à la place de l'hydroxyle en 3' du ribose - provoque l'arrêt de l'élongation de la chaîne d'ADN. L'ADN polymérase $\alpha$, responsable de la réplication de l'ADN nucléaire, n'a que peu d'affinité pour l'AZT, alors que la TI utilisera préférentiellement l'AZT au lieu de la thymidine. De nombreux tests cliniques ont démontré que les patients atteints du SIDA et traités à l'AZT ont une espérance de vie accrue par rapport aux patients non traités [18]. De plus, le traitement à l'AZT retarde l'apparition de symptômes chez les individus séropositifs asymptomatiques. Ce médicament provoque néanmoins des effets secondaires sérieux chez certains patients [19].

L'apparition de souches de HIV-1 résistantes à l'AZT chez certains individus représente une autre limite à l'efficacité de ce médicament. Les implications de cette résistance seront discutées plus loin.

Expression de l'ARNm et des protéines virales. Puisque la transcription et la traduction des gènes du virus nécessitent l'utilisation de la machinerie cellulaire, il n'y a aucun moyen propre d'inhiber les enzymes impliquées dans ces processus sans affecter l'expression des gènes cellulaires. Certaines approches relativement spécifiques du substrat de ces réactions sont cependant explorées. Par exemple, la ribavirine, efficace contre certains virus, est un analogue du ${ }^{7} \mathrm{MeG}-\mathrm{p}-\mathrm{p}-\mathrm{p}$ normalement ajouté à la partie 5' des ARNm. Cette substance est préférentiellement ajoutée aux extrémités des ARNm viraux nouvellement synthétisés et inhibe ainsi la synthèse des protéines virales. La ribavirine s'est de fait avérée efficace contre le HIV-1 dans des cellules en culture, mais sa très grande toxicité in vivo ne justifie pas son utilisation. De plus, des études ont démontré que la ribavirine et l'AZT, en association, peuvent avoir des effets antagonistes quant à leur capacité d'inhiber la réplication virale [20].

Assemblage et formation des virions. Le processus d'assemblage et de formation du bourgeon viral et l'abondance des protéines virales qui se trouvent à la surface des cellules infectées constituent des cibles potentielles sur lesquelles les interférons $\alpha$ et $\beta$ pourraient agir. En réalité, le mode d'action antivirale des interférons est incomplètement élucidé mais probablement multiple. Plusieurs travaux ont démontré que le traitement des lymphocytes infectés par le HIV-1 à l'interféron $\alpha$ recombinant provoque l'accumulation de virions défectueux dans le cytoplasme de ces cellules, sans toutefois réduire la viabilité de celles-ci [21, 22]. Des études cliniques sont en cours de réalisation.

\section{La résistance aux chimiothérapies}

Cinq types de mécanismes de résistance aux antibiotiques et aux chimiothérapies antivirales sont connus : (1) une diminution de la perméabilité de la membrane cellulaire à l'inhibiteur ; (2) une diminution ou perte d'une enzyme responsable de l'activation de l'inhibiteur ou une réduction spécifique de l'affinité entre l'activateur et l'inhibiteur; (3) une augmentation de la synthèse de l'enzyme touchée par l'inhibiteur ; (4) une réduction de l'affinité entre l'inhibiteur et l'enzyme cible et (5) une augmentation de la dégradation de l'inhibiteur, de ses métabolites ou cofacteurs.

La base de la résistance aux traitements chimiothérapeutiques des virus est la mutation du génome viral, induisant la synthèse de protéines altérées. Une telle mutation sera transmise aux générations suivantes 
du virus, conférant une stabilité du phénotype muté. Elle pourra ou non affecter la virulence ou le potentiel cytopathologique du virus ainsi que sa résistance ou sa sensibilité à d'autres agents chimiothérapeutiques. Les stratégies de chimiothérapie utilisées contre un virus doivent donc tenir compte non seulement du mécanisme de résistance développé par ce virus, mais aussi des changements phénotypiques de celui-ci. Par exemple, dans le cas de l'herpes simplex de type 1 (HSV-1), l'acyclovir, un inhibiteur de la synthèse de l'ADN viral, est utilisé pour limiter la réplication de ce virus. Au moins deux enzymes virales sont essentielles au métabolisme de cette drogue : la thymidine kinase (TK) virale, qui active l'acyclovir en triphosphate d'acyclovir et l'ADN-polymérase virale, qui incorpore l'acyclovir triphosphate dans la chaîne d'ADN en croissance. Des souches de HSV-1 résistantes ont été isolées de patients traités à l'acyclovir et de cellules en culture infectées, maintenues en présence d'acyclovir. Il fut démontré que ces souches résistantes avaient une virulence réduite et possédaient une mutation dans l'une ou l'autre des enzymes responsables du métabolisme de l'acyclovir, conférant à celles-ci une affinité réduite pour cette molécule [23].

L'importance de la dérive génétique du HIV-1 [6] et le taux élevé d'erreurs d'incorporation qu'introduit sa TI [24] rendaient prévisible l'isolement de mutants résistants à la zidovudine chez certains patients sous traitement. Deux groupes de recherche, dont le nôtre, ont effectivement isolé de tels mutants issus de patients atteints du SIDA, du CRS, complexe relié au SIDA ou ARC pour AIDSrelated complex, en anglais [9] ainsi que de patients séropositifs asymptomatiques subissant un traitement prolongé à la zidovudine [8]. Chacune des équipes impliquées a démontré qu'environ $75 \%$ des patients traités à la zidovudine développent un virus résistant à cette drogue. L'utilisation de surnageants de culture congelés, obtenus mensuellement, pour chacun des patients, tout au long du traitement, a permis à notre groupe d'établir qu'un minimum de 27 semaines est nécessaire à l'apparition de la $\mathrm{m} / \mathrm{s} n^{\circ} 2$, vol. 7, février 91

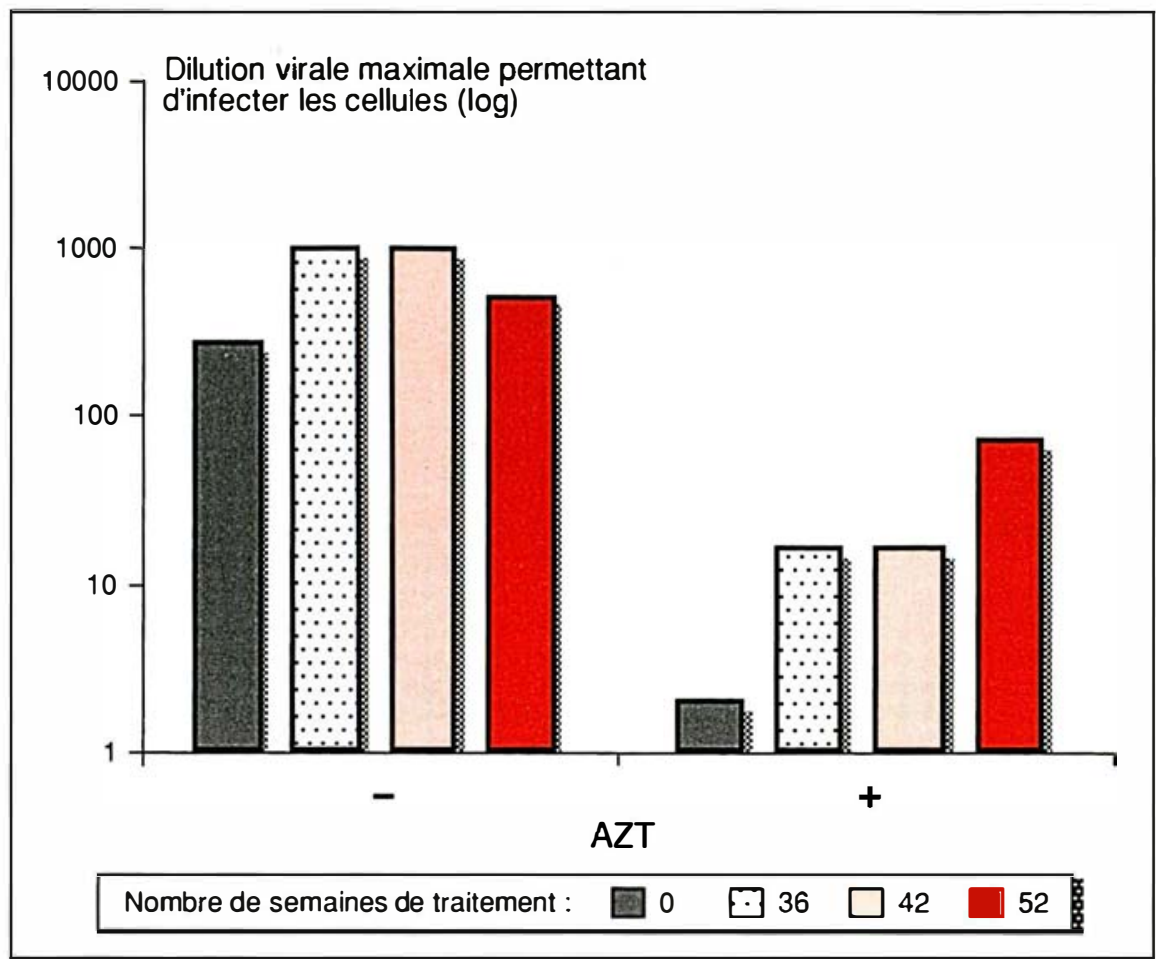

Figure 1. Adaptation graduelle d'une souche virale à la croissance en présence d'AZT. Quatre souches de HIV-1 ont été obtenues d'un même patient tout au long de sa thérapie à l'AZT. Une même quantité de virus, pour chacun des isolats étudiés, a été diluée en série (1:2) et incubée, en présence ou en absence d'AZT, avec des cellules sensibles à l'infection. Après 10 jours, la plus haute dilution à laquelle une souche virale pouvait établir une infection a été déterminée. En l'absence d'AZT, les quatre souches, même fortement diluées, peuvent infecter les cellules. En présence d'AZT, les dilutions maximales sont plus faibles et c'est le virus obtenu 52 semaines après le traitement du patient qui peut être utilisé à la dilution la plus élevée.

résistance à la zidovudine. L'examen plus quantitatif d'isolats séquentiels obtenus à partir d'un même patient montre l'adaptation graduelle d'une souche virale à la croissance en présence d'AZT. Dans l'expérience décrite (figure 1), cette adaptation se manifeste par une relation inverse entre la quantité de virus nécessaire à l'établissement d'une infection in vitro, réalisée en présence d'AZT, et le nombre de semaines écoulées entre le début du traitement à la zidovudine et le prélèvement de ce virus. En d'autres termes, la capacité d'une souche virale à croître en présence de drogue augmente avec le nombre de semaines de traitement du patient. Cliniquement, deux phénomènes, non exclusifs, peuvent expliquer ces résultats. D'abord, il est possible que la souche résistante représente une infime proportion de l'ensemble des variants du HIV-1 infectant initiale- ment le patient. Le traitement à la zidovudine provoquera alors une sélection positive de cet isolat, entraînant une augmentation progressive de sa prolifération chez le patient traité. L'infection in vitro de cellules sensibles, en présence d'AZT, sera donc plus rapide lorsqu'un tel virus est prédominant dans la circulation de l'individu infecté. Ces résultats peuvent également suggérer que plusieurs mutations sont nécessaires au développement d'une résistance totale à l'AZT et que chacun des changements génomiques, pris individuellement, ne confère qu'une résistance partielle à la drogue.

Le suivi de patients infectés par de telles souches a permis de montrer la stabilité du phénotype de résistance à l'AZT, puisque chacune des souches virales isolées postérieurement à l'isolement initial étaient elles-mêmes résistantes à l'AZT. A l'inverse, cer- 


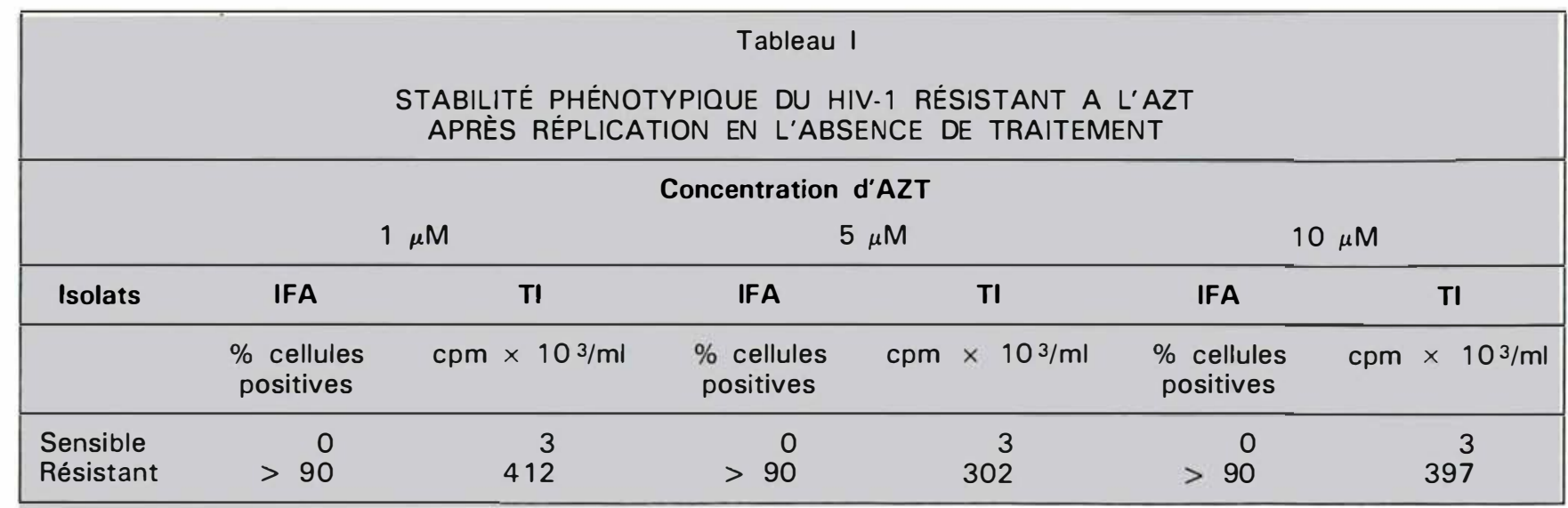

Un isolat résistant et sa contrepartie sensible à l'AZT ont été amplifiés en absence d'antiviral en culture de tissu, sur des cellules susceptibles. L'infection a été maintenue par l'addition régulière, dans chacune des cultures, de cellules non infectées. Après 14 jours, le surnageant clarifié de chacune des cultures a été ajouté à des cellules traitées à l'AZT (1,5 et 10 $\mu M$ ). Les cellules infectées ont été ensemencées à une concentration de $5 \times 10^{5}$ cellules/ml en présence d'AZT et incubées pour une période de 4 jours, après quoi l'activité de la transcriptase inverse (TI) et le pourcentage de cellules présentant des antigènes du HIV-1 à leur surface (IFA) ont été déterminés. Le premier paramètre consiste à mesurer l'incorporation par la $\mathrm{TI}$ de la $3[\mathrm{H}] \mathrm{dTTP}$ dans une molécule de poly(rA)-oligo-d(T) 12.18 après que ces produits ont été incubés en présence d'un lysat de virus contenant de la TI. Le deuxième paramètre identifie spécifiquement les cellules infectées par le HIV-1. Une suspension cellulaire, fixée sur une lamelle, est mise en présence d'un anticorps monoclonal dirigé contre une protéine spécifique du HIV-1 (p24). Cet anticorps est combiné à une molécule de fluorescéine qui émet une lumière visible après stimulation aux ultraviolets.

tains surnageants de culture obtenus avant l'isolement d'une souche virale résistante, contenaient eux aussi des mutants du HIV-1 résistants à la drogue. Il fut donc possible, pour quelques patients, d'identifier le moment précis à partir duquel chacun d'eux est devenu infecté par un virus résistant.

Des études effectuées in vitro sur de nombreux isolats résistants nous ont permis d'étendre nos connaissances d'une façon significative. Chaque virus mutant fut comparé au virus sensible à la zidovudine isolé à partir du même patient au début du traitement.

\section{Caractérisation biologique} des souches résistantes

La stabilité phénotypique décrite in vivo est également observée in vitro. En effet, les isolats résistants peuvent réinfecter, en présence du médicament, des cellules sensibles, même après avoir été propagés en culture de tissu en absence d'AZT pendant une période de 14 jours (Tableau I). De plus, l'utilisation d'ELISA quantitatifs, dirigés spécifiquement contre l'antigène p24 de la nucléocapside du virus et la TI ( $p 51 / 66)$, nous a permis d'établir que la relation stoechio- métrique entre la protéine p24 et la
TI est conservée entre les virus mutants et leur contrepartie sensible à l'AZT (résultat non montré). La résistance à l'AZT ne peut donc être associée à une plus grande quantité de TI par virion. Il s'ensuit que la mutation responsable de la résistance n'affecte pas la protéolyse du précurseur gag-pol nécessaire à la production des protéines tardives dont font partie TI et p24.

Puisque la zidovudine entre en compétition avec la désoxythymidine triphosphate (dTTP) au niveau du site actif de la TI, un changement d'affinité pour l'un ou l'autre de ces substrats pourrait expliquer la résistance à l'AZT. Ainsi, une plus grande affinité de l'enzyme mutante pour la dTTP aurait essentiellement les mêmes conséquences qu'une plus faible affinité pour la zidovudine: l'incorporation de drogue dans la chaîne naissante d'ADN serait diminuée et la réplication du virus serait, de ce fait, plus efficace.

Afin de vérifier ces hypothèses, le taux d'incorporation de la dTTP dans une molécule synthétique

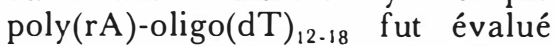
pour trois paires d'isolats sensibles et résistants. Chez deux patients sur les trois analysés (Tableau II), l'activité enzymatique $\left(\mathrm{V}_{\max }\right)$ de la protéine mutante est plus grande que celle de l'enzyme sauvage. En revanche, l'affinité $(\mathrm{Km})$ pour le nucléotide dTTP demeure inchangée. Il en résulte une augmentation d'un facteur 3 du rapport $\mathrm{V} / \mathrm{K}$ de l'activité enzymatique de la TI mutante par rapport à la TI normale. Ces résultats portent cependant sur un trop petit nombre de malades pour être extrapolés, d'autant que le rapport $\mathrm{V} / \mathrm{K}$ de l'isolat résistant du patient $B$ est semblable à celui de la souche de laboratoire sensible à l'AZT, le HIV-IIIb, et est nettement inférieur à celui de la souche sensible isolée du patient C. De plus, les souches résistantes et sensibles, isolées à partir de ce dernier, ont des activités enzymatiques similaires.

Dans un deuxième temps, l'affinité de la TI pour l'AZT fut évaluée pour les trois mêmes paires d'isolats. La concentration de médicament nécessaire à l'inhibition de chacune des TI mutantes est essentiellement la même que la concentration inhibitrice de chacune des souches sensibles correspondantes (Tableau III). Il n'existe également que peu ou pas de différences entre les concentrations de zidovudine nécessaires pour inhiber de $50 \%$ l'infection de cellules par un virus mutant ou par sa contrepartie sensible. Bref, in vitro, l'enzyme mutante semble avoir la 
Tableau II

COMPARAISON DE LA CINÉTIQUE D'INCORPORATION DU dTTP PAR DES TI RÉSISTANTES ET SENSIBLES A L'AZT

\begin{tabular}{|c|c|c|c|c|c|c|}
\hline \multirow[b]{2}{*}{$\begin{array}{c}\text { Origine } \\
\text { du HIV-1 étudié }\end{array}$} & \multicolumn{2}{|c|}{ Souches sensibles } & \multicolumn{4}{|c|}{ Souches résistantes } \\
\hline & $\begin{array}{c}\mathrm{Km} \\
(\mu \mathrm{M})\end{array}$ & $\begin{array}{c}V_{\text {max }} \\
\left(\mathrm{pmol} / 15^{\prime} / \mathrm{pg} 51 / 66\right)\end{array}$ & $\mathrm{V} / \mathrm{K}$ & $\begin{array}{l}\mathrm{Km} \\
(\mu \mathrm{M})\end{array}$ & $\underset{\left(\mathrm{pmol} / 15^{\prime} / \mathrm{mg} 51 / 66\right)}{V_{\text {ax }}}$ & $\mathrm{V} / \mathrm{K}$ \\
\hline Patient $A$ & 5,4 & 0.7 & 0,12 & 4.2 & 1.8 & 0,41 \\
\hline Patient B & 4,1 & 0.2 & 0.05 & 3,4 & 0,5 & 0,13 \\
\hline Patient C & 4,1 & 3,9 & 1,1 & 3,9 & 3,3 & 0,9 \\
\hline HIV-IIIb & 4,4 & 0,4 & 0,09 & $1^{*}$ & $1^{*}$ & $i^{*}$ \\
\hline
\end{tabular}

L'activité enzymatique de la $\mathrm{TI}\left(\mathrm{V}_{\mathrm{max}}\right)$ est définie comme étant le taux d'incorporation d'une molécule (substrat) dans un polymère synthétique d'acide nucléique dans des conditions saturantes de ce substrat. L'affinité de la $T I$ pour son substrat (Km) est la con-

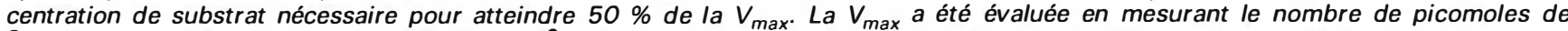
${ }^{3}$ [H]dTTP (activité spécifique $=225,2 \times 10^{8}$ (par/pmol) dans le poly(rA)-oligo(dT) $12-18$. Le Km représente $50 \%$ de la concentration de ${ }^{3}$ IHIdTTP à laquelle la $V_{\max }$ est atteinte. Les courbes ont été analysées à l'aide du logiciel Asystant Scientific Software IMacMillan Software, New York).

* : indéterminé

même affinité pour le dTTP et l'AZT qu'une enzyme sensible à la drogue. Ce dernier résultat suggère qu'au sein du virion d'importantes interactions entre protéines et acides nucléiques sont essentielles pour qu'une discrimination entre l'AZT et la dTTP puisse être faite par la TI mutante. La nature des expériences de cinétique et d'affinité décrites précédemment implique la solubilisation de la TI et donc la rupture de ces interactions. Les changements d'activité enzymatique entre protéines mutantes et sauvages (Tableau II) doivent donc être interprétés avec prudence. Néanmoins, la plupart des souches résistantes au médicament ont la capacité d'infecter des cellules sensibles en présence de $20 \mu \mathrm{M}$ d'AZT (résultat non montré). Cette concentration est au moins 200 fois supérieure à la concentration inhibitrice des souches sensibles à cette substance. De plus, la toxicité cellulaire de l'AZT à des concentrations supérieures à $20 \mu \mathrm{M}$ est telle que nous n'avons pu déterminer la concentration à laquelle les souches résistantes sont inhibées.

Toutefois, l'examen de la dose infectieuse en culture de tissu $\left(\right.$ TCID $\left._{50}\right)$ par rapport à une même quantité de protéines virales (p24) montre que les virus mutants sont moins infectieux que leur contrepartie sauvage, d'un

Tableau III

AFFINITÉ ET CONCENTRATION INHIBITRICE DE L'AZT SUR LES TI RÉSISTANTES ET SENSIBLES A L'AZT

\begin{tabular}{|c|c|c|c|c|}
\hline $\begin{array}{l}\text { Origine } \\
\text { du HIV-1 étudié }\end{array}$ & $\begin{array}{l}\text { Nombre de semaines après } \\
\text { le début du traitement }\end{array}$ & $\begin{array}{c}\text { Sensibilité de l'isolat } \\
\text { à l'AZT }\end{array}$ & $\begin{array}{c}\mathrm{K}_{\mathrm{i}} \\
(\mu \mathrm{M})\end{array}$ & $\begin{array}{l}{[\mathrm{ID}]_{50}} \\
(\mathrm{nM})\end{array}$ \\
\hline Patient $A$ & $\begin{array}{r}0 \\
52\end{array}$ & $\begin{array}{l}\text { sensible } \\
\text { résistant }\end{array}$ & $\begin{array}{l}7,2 \pm 1,6 \\
4,4 \pm 1,4\end{array}$ & $\begin{array}{l}28 \mathrm{nM} \\
17 \mathrm{nM}\end{array}$ \\
\hline Patient B & $\begin{array}{r}0 \\
28\end{array}$ & $\begin{array}{l}\text { sensible } \\
\text { résistant }\end{array}$ & $\begin{array}{l}6,0 \pm 1,1 \\
4,1 \pm 1,1\end{array}$ & $\begin{array}{l}20 \mathrm{nM} \\
13 \mathrm{nM}\end{array}$ \\
\hline Patient C & $\begin{array}{r}0 \\
32\end{array}$ & $\begin{array}{l}\text { sensible } \\
\text { résistant }\end{array}$ & $\begin{array}{r}18,3 \pm 8,1 \\
7,3 \pm 1,4\end{array}$ & $\begin{array}{l}47 \mathrm{nM} \\
18 \mathrm{nM}\end{array}$ \\
\hline Patient D & $\begin{array}{r}0 \\
82\end{array}$ & $\begin{array}{l}\text { sensible } \\
\text { résistant }\end{array}$ & $\begin{array}{l}6,6 \pm 1,7 \\
5,9 \pm 1,4\end{array}$ & $\begin{array}{l}18 \mathrm{nM} \\
18 \mathrm{nM}\end{array}$ \\
\hline HIV-IIIb & - & sensible & $5,9 \pm 1,6$ & ND* \\
\hline
\end{tabular}

Le $K_{i}$ est la moitié de la concentration à laquelle l'enzyme est complètement inhibée. La [ID] $]_{50}$ est la concentration de drogue qui inhibe l'infection de $50 \%$ des cellules. Les valeurs présentées ci-dessus ont été obtenues à différentes concentrations d'inhibiteur $(0,5,10,20,50,100$ et $200 \mathrm{nM})$ en utilisant comme substrat du 3(HITTP à $5 \mu \mathrm{M}$. Chaque expérience a été réalisée en duplicata. Les $K_{i}$ furent calculés en utilisant une équation pour l'inhibition compétitive et sont décrits ici comme moyennes \pm 1 écarttype standard pour chacune des 6 concentrations d'inhibiteur utilisées.

* : non déterminé.

$m / s n^{\circ} 2$, vol. 7 , février 91 
Tableau IV

COMPARAISON DE LA CAPACITÉ D'INFECTION D'ISOLATS DU HIV-1 RÉSISTANTS A LA ZIDOVUDINE

AVEC LEURS CONTREPARTIES SENSIBLES A CETTE DROGUE

\section{$\mathrm{TCID}_{50} / \mathrm{pg}$ p24 Ag}

Source du HIV-1 étudié

Souches sensibles

Souches résistantes

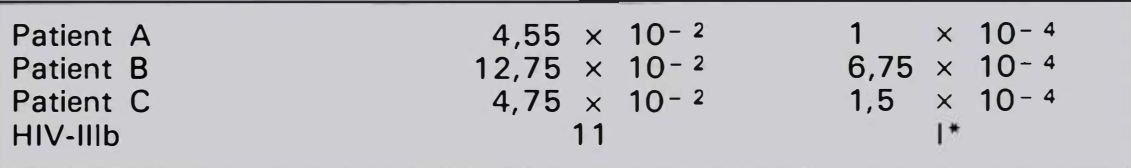

La capacité d'infection de chacune des souches virales étudiées a été déterminée sur des cellules de type MT-4 en absence de zidovudine. Une même quantité de chacune des souches étudiée $12000 \mathrm{pg}$ p24 ag) a été diluée en série (1:10) et incubée en présence de cellules MT-4 (50 000 cellules) pendant 4 jours. Les cellules ont ensuite été récoltées et l'expression de l'ag viral p24 à leur surface a été examinée. La TCID 50 est définie comme étant le logarithme négatif de la plus haute dilution de virus capable d'infecter $50 \%$ de cellules en culture.

* : indéterminable. facteur 100 environ (Tableau IV). Ces résultats sont confirmés par des expériences de mutagenèse dirigée faites sur le gène pol du HIV-1 [25]. En revanche, ces mêmes virus mutants causent une plus grande cytopathologie que les virus sensibles à l'AZT (Tableau V).

Ainsi, lorsqu'une quantité définie de virus résistant est mise en présence d'un nombre déterminé de cellules sensibles à l'infection par le HIV-1, un plus petit nombre $(1 / 100)$ de ces cellules n'est infecté que lorsque la souche parentale, sensible à la drogue, est utilisée pour une infection faite dans les mêmes conditions. Toutefois, la mortalité cellulaire est nettement plus rapide dans la culture de cellules mise en présence de la souche virale résistante que dans la culture où une souche sauvage est
Tableau $\mathrm{V}$

\begin{tabular}{|c|c|c|c|c|c|}
\hline $\begin{array}{r}\text { CY } \\
\text { DE CELLUL } \\
\text { RÉSISTAN }\end{array}$ & $\begin{array}{l}\text { Tableau } \\
\text { PATHOLOGIE ASSO } \\
\text { SUSCEPTIBLES PA } \\
\text { S A L'AZT ET LEUF }\end{array}$ & $\begin{array}{l}\text { EE A } \\
\text { DES } \\
\text { ONTF }\end{array}$ & $\begin{array}{l}\text { EECT } \\
\text { CHE } \\
\text { RTIE }\end{array}$ & NSIB & \\
\hline & & Pourc & $\begin{array}{l}\text { ge } \\
s \text { ap }\end{array}$ & $\begin{array}{l}\text { ellule } \\
\text { infec }\end{array}$ & iables \\
\hline $\begin{array}{l}\text { Source } \\
\text { du HIV-1 étudié }\end{array}$ & $\begin{array}{c}\text { Sensibilité } \\
\text { de I'isolat à I'AZT }\end{array}$ & 5 & 10 & 16 & 23 \\
\hline Patient A & $\begin{array}{l}\text { sensible } \\
\text { résistant }\end{array}$ & $\begin{array}{l}74 \\
70\end{array}$ & $\begin{array}{l}53 \\
58\end{array}$ & $\begin{array}{r}61 \\
5\end{array}$ & $\begin{array}{l}4 \\
0\end{array}$ \\
\hline Patient B & $\begin{array}{l}\text { sensible } \\
\text { résistant }\end{array}$ & $\begin{array}{l}74 \\
69\end{array}$ & $\begin{array}{l}56 \\
30\end{array}$ & $\begin{array}{r}62 \\
1\end{array}$ & $\begin{array}{l}3 \\
0\end{array}$ \\
\hline Patient C & $\begin{array}{l}\text { sensible } \\
\text { résistant }\end{array}$ & $\begin{array}{l}68 \\
69\end{array}$ & $\begin{array}{l}50 \\
40\end{array}$ & $\begin{array}{r}61 \\
3\end{array}$ & $\begin{array}{r}26 \\
0\end{array}$ \\
\hline Aucun virus & - & 84 & 74 & 67 & 60 \\
\hline
\end{tabular}

Les souches virales (2000 pg) ont été incubées pour une période de 3 heures en présence de $1 \times 10^{6}$ cellules MT-4. Les cellules ont ensuite été ensemencées à une densité de $3 \times 10^{5}$ cellules $/ \mathrm{ml}$ et incubées en absence d'AZT. Le pourcentage de cellu- utilisée. En d'autres termes, malgré l'apparente difficulté d'infection d'une cellule par une souche résistante, une fois infectée, cette cellule sera détruite beaucoup plus rapidement que lorsqu'elle est infectée par une souche sensible au médicament. Ces résultats contrastent avec ce qui est connu des souches HSV-1 résistantes à l'acyclovir [23]. En effet, toutes les souches HSV-1 résistantes à l'acyclovir, isolées et caractérisées jusqu'à ce jour, ont une cytopathologie réduite par rapport à celle des souches sauvages. De plus, les isolats mutants se répliquent plus lentement que les isolats sauvages. Finalement, des études in vitro ont démontré que, pour HSV-1, la source de résistance est un changement d'affinité de l'enzyme cible pour l'acyclovir.

\section{Résistance et susceptibilité à d'autres nucléosides}

L'efficacité de l'AZT en essai clinique a motivé le développement de plusieurs autres analogues de nucléosides qui ont également comme effet d'inhiber la TI. Certains de ces composés, notamment la didésoxyinosine (DDI) et la didésoxycytidine (DDC), font l'objet d'études cliniques en phase I. Nous pensons que le développement et l'utilisation clinique de l'une ou l'autre de ces substances devraient non seulement tenir compte de leur synergie potentielle avec l'AZT, mais également assurer que la possibilité de double résistance d'un même virus aux produits utilisés est inexistante.

Il nous est donc apparu important de déterminer la sensibilité des isolats AZT-résistants à un certain nombre de ces nouveaux composés. Dans chacun des cas étudiés, la concentration de drogue nécessaire à l'inhibition de la souche résistante à l'AZT fut comparée à celle nécessaire à l'inhibition de la souche sauvage provenant du même patient. Un exemple d'une souche résistante à la fois à l'AZT et au D4T (2'3' didehydro, 2'3' didesoxythymidine) est montré sur la figure 2. Cette souche nécessite dix fois plus d'AZT et trente fois plus de D4T qu'une souche sensible pour être inhibée au même niveau. Les résultats d'une autre équipe sug- 
gèrent que les souches de HIV-1 résistantes à l'AZT sont également résistantes à tout nucléoside possédant un groupe azido $\left(\mathrm{N}_{3}\right)$ lié au carbone 3' de son ribose et sont aussi sensibles à tout autre nucléoside [26]. Ainsi, ce groupe de chercheurs associe la résistance à l'AZT à un changement de conformation dans la structure tridimensionelle de la TI qui empêcherait tout nucléotide pos- sédant un groupe azido d'entrer dans le site actif de l'enzyme et donc d'être incorporé dans la molécule d'ADN. Sans être mutuellement exclusifs, nos résultats semblent en contradiction partielle avec cette hypothèse. En effet, nous .avons trouvé que certains virus résistants à l'AZT peuvent avoir une résistance croisée avec des composés autres que ceux possédant un groupe azido.
Nous pouvons voir (figure 2) que les courbes d'inhibition d'infection d'une souche sensible et d'une souche résistante à l'AZT sont identiques lorsque deux analogues de la cytidine sont utilisés (DDC et DTC). En revanche, lorsqu'un analogue de la thymidine autre que la zidovudine est utilisé, une résistance croisée est notée. La souche sauvage, sensible à la zidovudine, montre la même courbe d'inhi-

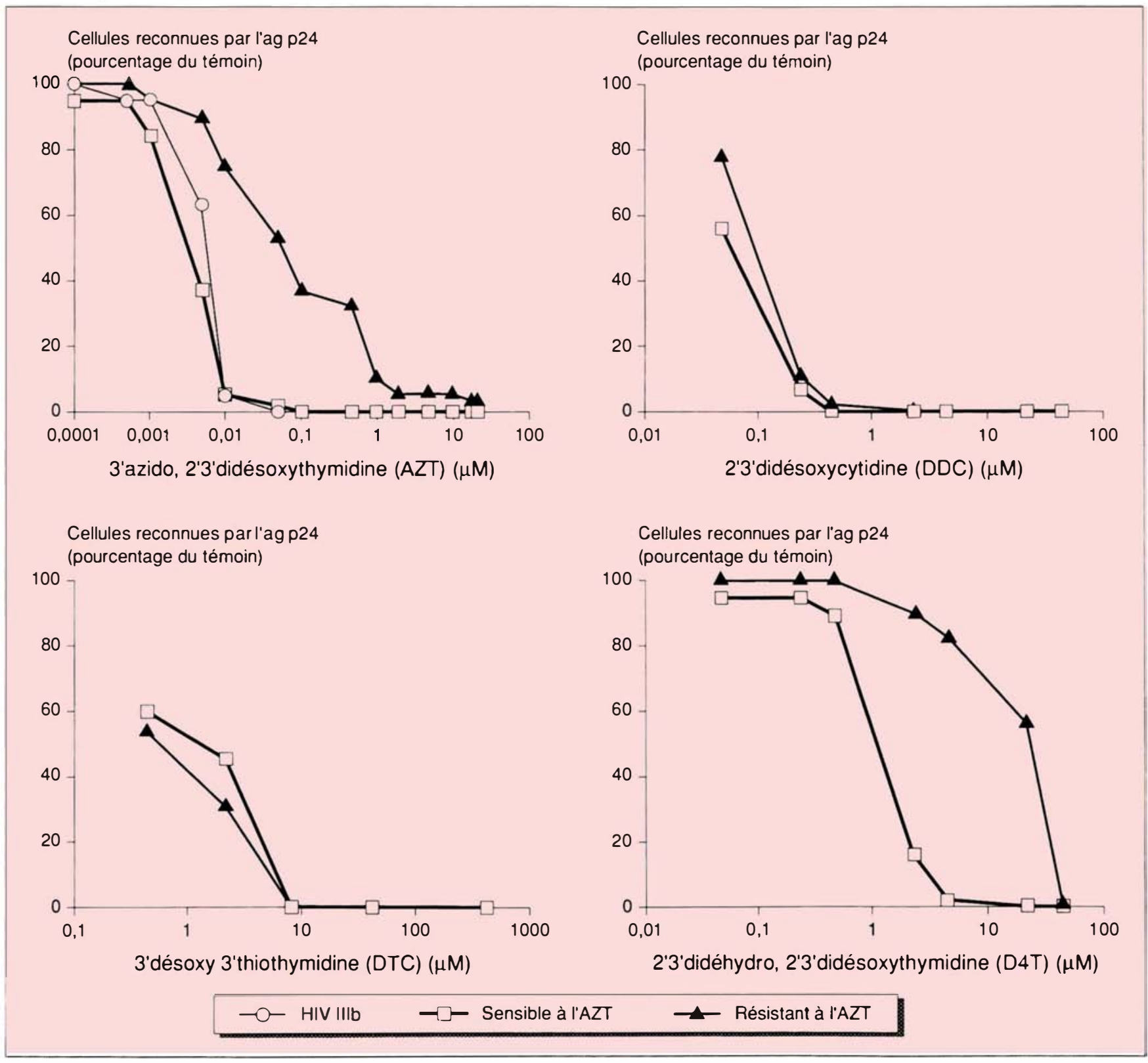

Figure 2. Corésistance d'un isolat à la fois résistant à la zidovudine et au D4T. Une même quantité de virus résistant ou sensible à l'AZT a été ajoutée à un nombre de cellules sensibles en présence de diverses concentrations des quatre inhibiteurs. Le pourcentage de cellules reconnues par un anticorps anti-p24 spécifique du HIV-1 a été déterminé. Le résultat est exprimé en pourcentage d'une infection produite en absence d'agent antiviral. 


\section{RÉFÉRENCES}

17. Furman PA, Fyfe JA, Saint Clair $M H$, el al. Phosphorylation of 3'-azido-3'deoxythymidine and selective interaction of the 5'-triphosphate with HIV reverse transcriptase. Proc Nall Acad Sci USA 1986 ; 83 8333-7.

18. Fischl MA, Richman DD, Grieco $\mathrm{MH}$ et al. The efficacy of azidothymidine (AZT) in the treatment of patients with AIDS and AIDS-related complex. $N$ Engl J Med 1987 ; 317 : 185-91

19. Richman DD, Fischl MA, Grieco $M H$, et al. The toxicity of azidothymidine (AZT) in the treatment of patients with AIDS and AIDS-related complex. $N$ Engl J Med 1987 ; 317 : 192-7.

20. Vogt MW, Hartshorn KL, Furman PA, el al. Ribavirin antagonizes the effect of azidothymidine on HIV replication Science 1987 ; 235 : 1376-9.

21. Poli G, Orenstein JM, Kinter S, el al. Interferon- $\alpha$ but not AZT suppresses HIV expression in chronically infected cell lines. Science 1989 ; 244 : 575-7.

22. DeWit R, Boucher CA, Veenhof KHN Clinical and virological effects of high-dose recombinant interferon- $\alpha$ in disseminated AIDS-related Kaposi's sarcoma. Lancel 1988 ; i : 1214-7.

23. Larder BA, Darby G. Virus drug resistance: mechanisms and consequences. Antiviral Res 1984; 4 : 1-42.

24. Roberts JD, Bebenek K, Kunkel TA. The accuracy of reverse transcriptase from HIV-1. Science 1988; 242 : 1171-4

25. Larder BA, Kemp SD, Punifay DJM Infectious potential of human immunodeficiency virus type 1 reverse transcriptase mutants with altered inhibition sensitivity. Proc nall Acad Sci USA 1989 ; 86 : 4803-7.

26. Larder BA, Chesebro B, Richman DD. Susceptibilities of zidovudine-susceptible and-resistant human irnmunodeficiency virus isolates to antiviral agents determined by using a quantitative plage reduction assay. Antimicrobiol Agents Chemolher 1990; 34 436-41.

27. Larder BA, Kemp SD. Multiple mutations in HIV-1 reverse transcriptase confer high-level resistance to zidovudine (AZT). Science $1989 ; 246$ : 1155-8.

\section{Remerciements}

Ces travaux bénéficient du soutien financier de Santé et Bien-être social Canada et ont été réalisés dans le cadre d'une étude prédoctorale menée par Ronald Rooke, récipiendaire d'une bourse de la Société. canadienne de recherche sur le cancer. Les auteurs remercient Stéphan Bour, Louis Mercure et Hugo Soudeyns pour leurs commentaires scientifiques, ainsi que Rhona Rosenzweig et Sandy Fraiberg pour la dactylographie. bition que celle de la souche de laboratoire HIV-IIIb. En revanche, comparée à une souche sensible, la souche résistante à la drogue requiert une concentration d'AZT 80 fois plus élevée pour atteindre le même niveau d'inhibition. En ce qui concerne la résistance croisée, l'inhibition d'une souche résistante requiert 30 fois plus de D4T que celle de la souche sensible.

La caractérisation moléculaire de quelques isolats AZT-résistants a été publiée récemment [27]. Trois mutations particulières spécifiques du gène pol confèrent un degré élevé de résistance à l'AZT lorsqu'elles sont associées. Une quatrième mutation peut également être présente dans certains cas. Il s'agit des substitutions Asp ${ }^{67} \rightarrow$ Asn, Lys ${ }^{70} \rightarrow$ Arg, Thr ${ }^{215}$ $\rightarrow$ Phe ou Tyr et Lys ${ }^{219} \rightarrow$ Gln. Différentes combinaisons de ces mutations peuvent engendrer des niveaux intermédiaires de résistance. L'analyse de la séquence nucléotidique des mutants ayant une résistance croisée à une ou plusicurs autres drogues montrera sans doute, en plus des changements nécessaires à la résistance à l'AZT décrits plus haut, d'autres changements permettant à l'enzyme mutante de discriminer entre l'analogue et le nucléotide correspondant. Nos résultats indiquent que le traitement de sidéens à la zidovudine n'empêche pas totalement la réplication du HIV-1. Le taux de réplication résiduel semble faciliter l'évolution du virus vers la résistance. Il est donc impérieux que d'autres agents chimiothérapeutiques soient développés et utilisés en association avec l'AZT. Ainsi, non seulement la réplication du HIV-1 serait réduite à un minimum, mais il serait presque impossible pour un isolat particulier de devenir résistant aux deux (ou plus) médicaments à la fois. Pour ce faire, il est toutefois essentiel de choisir comme second agent thérapeutique une molécule dont les caractéristiques minimiseraient les chances de développement de résistance croisée. L'isolement de nouvelles souches résistantes à la zidovudine permet de tester de nouveaux agents inhibiteurs de la réplication du HIV-1, et ainsi de sélectionner de nouvelles substances thérapeutiques sans résistance croisée avec l'AZT

\section{Summary}

Isolation of HIV-1 strains resistant to AZT : limitations in the use of nucleoside analogs in the treatment of AIDS

An increasing number of antiviral strategies have been developed in the war against the human immunodeficiency virus (HIV-1). However, only one compound, 3'azido-3'-deoxythymidine (AZT or zidovudine) is being used extensively in the treatment of AIDS. Unfortunately, about $75 \%$ of AZT-treated patients can be expected to develop drug-resistant strains of HIV -1 . These variants can infect susceptible cells in the presence of AZT concentration 100 times higher than those inhibitory to wild-type strains. Results on the affinity of certain nuclcotides or their analogs for viral reverse transcriptase (RT) as well as the RT activity of the resistant viruses did not differ significantly from those obtained when the wild-type viruses were studied in in vitro assays. The resistant strains studicd where shown to be more cytopathic although less infectious than corresponding AZT-sensitive strains obtained from the same patients at times prior to commencement of therapy. The mutations responsible for the change in phenotype have been characterized and shown to occur within the pol region of the viral genome. Certain AZTresistant isolates show crossresistance to other nucleoside analogs that have potential clinical value. Finally, resistance to AZT is discussed in the context of other anti-HIV-1 strategics.

\section{TIRÉS A PART}

M.A. Wainberg 